\title{
Uniformly supported sets and fixed points properties
}

\section{ANDrei Alexandru and Gabriel Ciobanu}

\begin{abstract}
.
The theory of finitely supported algebraic structures is a reformulation of Zermelo-Fraenkel set theory in which every set-based construction is finitely supported according to a canonical action of a group of permutations of some basic elements named atoms. In this paper we study the properties of finitely supported sets that contain infinite uniformly supported subsets, as well as the properties of finitely supported sets that do not contain infinite uniformly supported subsets. Particularly, we focus on fixed points properties.
\end{abstract}

Acknowledgements. The authors are grateful to the anonymous referees for their useful comments and suggestions which improve the paper.

\section{REFERENCES}

[1] Alexandru, A. and Ciobanu, G., Finitely Supported Mathematics: An Introduction, Springer, 2016

[2] Alexandru, A. and Ciobanu, G., Fuzzy sets within Finitely Supported Mathematics, Fuzzy Sets and Systems 339, 2018, 119-133

[3] Alexandru, A. and Ciobanu, G., Finitely Supported Sets Containing Infinite Uniformly Supported Subsets. Proceedings Third Symposium on Working Formal Methods, Electronic Proceedings in Theoretical Computer Science, vol. 303, 2019, 120-134

[4] Alexandru, A. and Ciobanu, G., Fixed point results for finitely supported algebraic structures, Fuzzy Sets and Systems, 2019 (in press) available online https:/ / doi.org/10.1016/j.fss.2019.09.014

[5] Bojanczyk, M., Klin, B. and Lasota, S., Automata with group actions, in Proceedings of the 26th Symposium on Logic in Computer Science, IEEE Computer Society Press, 2011, 355-364

[6] Pitts, A. M., Nominal Sets Names and Symmetry in Computer Science, Cambridge University Press, 2013

Romanian ACADEMY, InStitute OF COMPUTER SCIENCE, IAŞI

A. I. CUZA UNIVERSITY OF IAŞI, ROMANIA

Email address: andrei.alexandrueiit.academiaromana-is.ro

Email address: gabrieleinfo.uaic.ro

Received: 16.04.2019; In revised form: 09.01.2020; Accepted: 16.01.2020

2010 Mathematics Subject Classification. 03E30, 03E25, 03E35, $03 B 70$.

Key words and phrases. fixed point, finite support, atomic (finite) powerset, uniform support.

Corresponding author: Gabriel Ciobanu; gabriel@info.uaic.ro 